\title{
AN ASYMPTOTIC EXPANSION FOR THE EXPECTED NUMBER OF REAL ZEROS OF A RANDOM POLYNOMIAL
}

\author{
J. ERNEST WILKINS, JR.
}

(Communicated by William D. Sudderth)

\begin{abstract}
Let $\nu_{n}$ be the expected number of real zeros of a polynomial of degree $n$ whose coefficients are independent random variables, normally distributed with mean 0 and variance 1 . We find an asymptotic expansion for $\nu_{n}$ of the form

$$
\nu_{n}=\frac{2}{\pi} \log (n+1)+\sum_{p=0}^{\infty} A_{p}(n+1)^{-p},
$$

in which $A_{0}=0.625735818, A_{1}=0, A_{2}=-0.24261274, A_{3}=0, A_{4}=$ $-0.08794067, A_{5}=0$. The numerical values of $\nu_{n}$ calculated from this expansion, using only the first four, or six, coefficients, agree with previously tabulated seven decimal place values $(1 \leq n \leq 100)$ with an error of at most $10^{-7}$ when $n \geq 30$, or $n \geq 8$.
\end{abstract}

1. Introduction. Suppose that the coefficients of the polynomial $f(z)=a_{0} z^{n}+$ $a_{1} z^{n-1}+\cdots+a_{n}$ are independent, normally distributed random variables with mean 0 and variance 1 . Kac [1] has shown that the expected number of real zeros of $f(z)$ is

$$
\nu_{n}=\frac{4}{\pi} \int_{0}^{1} \frac{\left\{1-h_{n}^{2}(x)\right\}^{1 / 2}}{1-x^{2}} d x,
$$

in which $h_{n}(x)=(n+1) x^{n}\left(1-x^{2}\right) /\left(1-x^{2 n+2}\right)$. Kac [1] also showed that $\nu_{n} \sim$ $(2 / \pi) \log n$ for large $n$. Jamrom $[2,3]$ improved this result and showed that

$$
\begin{gathered}
\lim _{n=\infty}\left\{\nu_{n}-(2 / \pi) \log (n+1)\right\}=A_{0}, \\
A_{0}=\frac{2}{\pi}\left\{\log 2+\int_{0}^{1}\left(1-t^{2} \operatorname{csch}^{2} t\right)^{1 / 2} t^{-1} d t\right. \\
\left.-\int_{1}^{\infty}\left\{1-\left(1-t^{2} \operatorname{csch}^{2} t\right)^{1 / 2}\right\} t^{-1} d t\right\} .
\end{gathered}
$$

Wang [4] also derived (2); his value of $A_{0}$ is

$$
\frac{8}{\pi} \int_{0}^{1}\left(1-y^{2}\right)^{-1}\left\{1+\frac{\left(1-y^{2}-2 y \log y\right)^{1 / 2}}{\left(1-y^{2}+2 y \log y\right)^{1 / 2}}\right\}^{-1} d y
$$

(The substitution $y=e^{-t}$ and some simple manipulations show that the two values of $A_{0}$ are the same.)

Received by the editors May 23, 1987.

1980 Mathematics Subject Classification (1985 Revision). Primary 60G99.

(C) 1988 American Mathematical Society $0002-9939 / 88 \$ 1.00+\$ .25$ per page 
Several authors $[1,4-8]$ have found upper and/or lower bounds for $\nu_{n}$, all of which have been superseded, or anticipated, by the result of $\mathrm{Yu}$ [9] that $\nu_{n}-$ $(2 / \pi) \log (n+1)$ is an increasing sequence, so that

$$
0.558728799<\nu_{1}-(2 / \pi) \log 2 \leq \nu_{n}-(2 / \pi) \log (n+1)<A_{0} .
$$

In this paper we will prove the following result.

THEOREM. There is an asymptotic expansion of the form

$$
\nu_{n} \sim(2 / \pi) \log (n+1)+\sum_{p=0}^{\infty} A_{p}(n+1)^{-p}
$$

in which $A_{0}$ is defined by (3), $A_{1}=A_{3}=A_{5}=0$, and

$$
\begin{aligned}
& A_{2}=-\frac{1}{3 \pi} \int_{0}^{\infty}\left\{\left(1-t^{2} \operatorname{csch}^{2} t\right)^{-1 / 2}-1\right\} t d t \\
& A_{4}=-\frac{1}{180 \pi} \int_{0}^{\infty}\left\{7-12\left(1-t^{2} \operatorname{csch}^{2} t\right)^{-1 / 2}+5\left(1-t^{2} \operatorname{csch}^{2} t\right)^{-3 / 2}\right\} t^{3} d t
\end{aligned}
$$

The discovery that $A_{1}=A_{3}=A_{5}=0$ was unexpected. (We have been unable to decide the irresistible conjecture that $A_{p}=0$ for all odd $p$.) We note that $A_{0}=0.625735818, A_{2}=-0.24261274, A_{4}=-0.08794067$. Our numerical value for $A_{0}$ does not agree with the value 0.6312 reported by Wang [4].

2. Preliminaries. In this section we perform certain algebraic manipulations useful for the subsequent analysis. The variable $x$ is a number on the interval $(0,1)$, and $n$ and $N-1$ are positive integers. It is convenient to assume that $n \geq 8$.

We define $\delta, a, t, w$ and $\mu$ so that $\delta=1-(n+1)^{-1 / 2}, a=(n+1)^{1 / 2}, x=$ $1-t /(n+1), x^{n}=e^{w-t}, x^{2 n+2}=e^{-\mu-2 t}$. Then $\delta \geq 2 / 3, a \geq 3,0 \leq t \leq a$ when $\delta \leq x \leq 1, w=t+n \log x, \mu=-2 t-(2 n+2) \log x$. It is now easy to verify the results stated in the following lemma.

LEMMA 1. If $B, C, D$ and $E$ are defined so that

$$
\begin{aligned}
& B=e^{2 w}-1, C=t / 2(n+1), D=\left(1-e^{-\mu}\right) /\left(e^{2 t}-1\right) \\
& E=\frac{\left(2 D-B+2 C+D^{2}-C^{2}+2 B C-B C^{2}\right) t^{2} \operatorname{csch}^{2} t}{(1+D)^{2}\left(1-t^{2} \operatorname{csch}^{2} t\right)}
\end{aligned}
$$

then

$$
\begin{gathered}
h_{n}^{2}(x)=4 t^{2} e^{-2 t}(1+B)(1-C)^{2}\left(1-e^{-2 t}\right)^{-2}(1+D)^{-2} \\
1-h_{n}^{2}(x)=\left(1-t^{2} \operatorname{csch}^{2} t\right)(1+E)
\end{gathered}
$$

According to Taylor's Theorem, there is a number $\theta_{1}$, depending on $x$ and $N$, such that $0<\theta_{1}<1$ and

$$
\log x=-\sum_{p=1}^{N} p^{-1} t^{p}(n+1)^{-p}-(N+1)^{-1} t^{N+1}\left(n+1-\theta_{1} t\right)^{-N-1} .
$$

Because $n+1 \geq n+1-\theta_{1} t \geq n+1-a \geq 2(n+1) / 3$ when $0 \leq t \leq a$ and $n \geq 8$, the number $v_{N}$ defined as $\left\{(n+1) /\left(n+1-\theta_{1} t\right)\right\}^{N+1}$ is such that $1 \leq v_{N} \leq(3 / 2)^{N+1}$. It is now easy to see that the following lemma is true. 
LEMMA 2. If $\psi_{p N}(t)$ and $\phi_{p N}(t)$ are defined so that

$$
\begin{gathered}
\psi_{p N}=2 /(p+1), \quad \phi_{p N}=(p+1-p t) / p(p+1) \quad \text { when } p=1,2, \ldots, N-1, \\
\psi_{N N}=2 v_{N} /(N+1), \quad \phi_{N N}=N^{-1}-n v_{N} t /(N+1)(n+1),
\end{gathered}
$$

then

$$
\mu=t \sum_{p=1}^{N} \psi_{p N}(t) t^{p}(n+1)^{-p}, \quad w=\sum_{p=1}^{N} \phi_{p N}(t) t^{p}(n+1)^{-p}
$$

We furnish upper bounds for $w$ and $e^{2 w}$ in the following lemma.

LEMMA 3. If $0 \leq x \leq 1$ and $n \geq 8$, then $w \leq 1-8 \log (9 / 8)<0.05774$, and $e^{2 w} \leq(8 / 9)^{16} e^{2}<1.1225$.

If we consider $w=(n+1)(1-x)+n \log x$ as a function of $x$ on $(0,1)$, we see that $d w / d x=-n-1+n x^{-1}, d^{2} w / d x^{2}=-n x^{-2}<0$. It follows that $w$ attains its absolute maximum $w_{\max }$ when $x=n /(n+1)$, and that

$$
w_{\max }=1+n \log \left\{1-(n+1)^{-1}\right\}=\sum_{p=1}^{\infty} \frac{1}{p(p+1)(n+1)^{p}} .
$$

Hence $w_{\max }$ is a decreasing function of $n$. This remark and some arithmetic suffice to prove the lemma.

We can now find a lower bound for $E$.

LEMMA 4 . If $\delta \leq x \leq 1$ and $n \geq 8$, then $E \geq-0.6508$.

It follows from Lemma 2 when $\delta \leq x \leq 1$ and $n \geq 8$ that

$$
\begin{gathered}
0 \leq \frac{t^{2}}{n+1} \leq \mu=\frac{t^{2}}{n+1}\left\{1+\frac{2 v_{2} t}{3(n+1)}\right\} \leq \frac{t^{2}}{n+1}\left\{1+\frac{9 t}{4(n+1)}\right\} \\
2 D \geq\left(2 \mu-\mu^{2}\right) /\left(e^{2 t}-1\right) .
\end{gathered}
$$

Moreover, $B=e^{2 w}-1=2 w+2 w^{2} \exp \left(2 \theta_{2} w\right)$ for some $\theta_{2}$ such that $0<\theta_{2}<1$. It then follows from Lemma 3 that

$$
2 w \leq B \leq 2 w+2 w^{2} \exp \{\max (2 w, 0)\} \leq 2 w+2.245 w^{2} .
$$

We infer from the definitions of $w$ and $t$ that

$$
\begin{aligned}
(n+1)^{-1} & =1-n(n+1)^{-1} \geq \frac{w}{t}=1-\sum_{p=1}^{\infty} n p^{-1} t^{p-1}(n+1)^{-p} \\
& \geq 1-\sum_{p=1}^{\infty} n p^{-1} a^{-p-1}=-\frac{1}{2 a}+\sum_{p=2}^{\infty} \frac{2}{\left(p^{2}-1\right) a^{p}}
\end{aligned}
$$

Hence $w \leq t /(n+1),-1 / 2 a \leq w / t \leq 1 / 2 a, w^{2} \leq t^{2} / 4(n+1)$. 
If $R=2 D-B+2 C+D^{2}-C^{2}+B C(2-C)$, we now see that

$$
\begin{aligned}
-R \leq & -\frac{2 \mu-\mu^{2}}{e^{2 t}-1}+\left(2 w+2.245 w^{2}\right)-\frac{t}{n+1}-0 \\
& +\frac{t^{2}}{4(n+1)^{2}}-\frac{2 w t}{n+1}\left\{1-\frac{t}{4(n+1)}\right\} \leq-\frac{2 t^{2}}{(n+1)\left(e^{2 t}-1\right)} \\
& +\frac{t^{4}}{(n+1)^{2}\left(e^{2 t}-1\right)}\left\{1+\frac{9 t}{2(n+1)}\right\}+\frac{81 t^{2}}{16(n+1)^{2}} \\
& +\frac{2 t}{n+1}+\frac{0.56125 t^{2}}{n+1}-\frac{t}{n+1}+\frac{t^{2}}{4(n+1)^{2}} \\
& +\frac{t^{2}}{(n+1)^{3 / 2}}=t^{2}(n+1)^{-1}\left\{P_{0}(t)+(n+1)^{-1 / 2}+P_{1}(t)(n+1)^{-1}\right. \\
& \left.\quad+P_{2}(t)(n+1)^{-2}+P_{3}(t)(n+1)^{-3}\right\}
\end{aligned}
$$

in which

$$
\begin{gathered}
P_{0}(t)=t^{-1}-2\left(e^{2 t}-1\right)^{-1}+0.56125, \quad P_{1}(t)=t^{2}\left(e^{2 t}-1\right)^{-1}+\frac{1}{4} \\
P_{2}(t)=4.5 t^{3}\left(e^{2 t}-1\right)^{-1}, \quad \text { and } \quad P_{3}(t)=5.0625 t^{4}\left(e^{2 t}-1\right)^{-1} .
\end{gathered}
$$

It is a straightforward task to estimate the maxima of the functions $P_{l}(t)$. We find that $P_{0}(t) \leq 1.56125, P_{1}(t) \leq 0.4120, P_{2}(t) \leq 0.7996, P_{3}(t) \leq 1.5124$. Because $n \geq$ 8 and $D \geq 0$, it follows that $-R \leq 1.9524 t^{2}(n+1)^{-1},-E \leq 1.9524 \chi(t)(n+1)^{-1}$, in which

$$
\chi(t)=t^{4}\left(1-t^{2} \operatorname{csch}^{2} t\right)^{-1} \operatorname{csch}^{2} t=t^{4} /\left(\sinh ^{2} t-t^{2}\right) .
$$

The validity of Lemma 4 is now a consequence of the inequality, $\sinh ^{2} t-t^{2} \geq t^{4} / 3$.

There is a number $\theta_{3}$ such that $0<\theta_{3}<1$ and

$$
B=e^{2 w}-1=\sum_{k=1}^{N} \frac{2^{k} b_{k N} w^{k}}{k !},
$$

in which $b_{k N}=1$ when $k=1,2, \ldots, N-1$, and $b_{N N}=\exp \left(2 \theta_{3} w\right)<1.1225$. It now follows from Lemma 2 that

$$
B=\sum_{p=1}^{N^{2}} \beta_{p N}(t) t^{p}(n+1)^{-p},
$$

in which $\beta_{p N}$ is a polynomial of degree $p$ in $t$, whose coefficients are independent of both $n$ and $N$, when $1 \leq p \leq N-1$, and $\beta_{p N}$ is bounded when $0 \leq t \leq a$ by a polynomial of degree $N$ in $t$, whose coefficients are independent of $n$, when $N \leq p \leq N^{2}$. Therefore,

$$
B=\sum_{p=1}^{N} B_{p N}(t) t^{p}(n+1)^{-p}
$$

in which $B_{p N}=\beta_{p N}$ when $1 \leq p \leq N-1$, and

$$
B_{N N}=\sum_{p=N}^{N^{2}} \beta_{p N} t^{p-N}(n+1)^{-p+N}
$$


so that $B_{N N}$ is bounded when $0 \leq t \leq a$ by a polynomial of degree $N$ in $t$ whose coefficients are independent of $n$. In particular,

$$
\begin{aligned}
& B_{1 N}=2-t, \quad B_{2 N}=\frac{1}{6}\left(18-16 t+3 t^{2}\right), \quad B_{3 N}=\frac{1}{6}\left(24-29 t+10 t^{2}-t^{3}\right), \\
& B_{4 N}=\frac{1}{360}\left(1800-2664 t+1280 t^{2}-240 t^{3}+15 t^{4}\right), \\
& B_{5 N}=\frac{1}{360}\left(2160-3708 t+2224 t^{2}-590 t^{3}+70 t^{4}-3 t^{5}\right)
\end{aligned}
$$

when $N \geq 6$.

In a similar manner we find that

$$
D=\omega(t) \sum_{p=1}^{N} D_{p N}(t) t^{p}(n+1)^{-p}
$$

in which $\omega(t)=t /\left(e^{2 t}-1\right)=t(\operatorname{coth} t-1) / 2$, and $D_{p N}$ is a polynomial of degree $p-1$ in $t$, whose coefficients are independent of $n$ and $N$, when $1 \leq p \leq N-1$, and $D_{N N}$ is bounded when $0 \leq t \leq a$ by a polynomial of degree $N-1$ in $t$ whose coefficients are independent of $n$. In particular,

$$
\begin{gathered}
D_{1 N}=1, \quad D_{2 N}=\frac{1}{6}(4-3 t), \quad D_{3 N}=\frac{1}{6}\left(3-4 t+t^{2}\right) \\
D_{4 N}=\frac{1}{360}\left(144-260 t+120 t^{2}-15 t^{3}\right) \\
D_{5 N}=\frac{1}{360}\left(120-264 t+170 t^{2}-40 t^{3}+3 t^{4}\right.
\end{gathered}
$$

when $N \geq 6$. It follows that

$$
R=\sum_{p=1}^{N} R_{p N}(t) t^{p}(n+1)^{-p}
$$

in which $R_{p N}$ is independent of $n$ and $N$ and is bounded when $0 \leq t<\infty$ by a polynomial of degree $p$ in $t$ when $1 \leq p \leq N-1$. Also, $R_{N N}$ is bounded when $0 \leq t \leq a$ by a polynomial of degree $N$ in $t$ whose coefficients are independent of n. In particular,

$$
\begin{aligned}
R_{1 N}= & 2 \omega-1+t=t\left(\operatorname{coth} t-t^{-1}\right), R_{2 N}=\omega^{2}+\frac{1}{3}(4-3 t) \omega-\frac{1}{12}\left(15-20 t+6 t^{2}\right) \\
R_{3 N}= & \frac{1}{3}(4-3 t) \omega^{2}+\frac{1}{3}\left(3-4 t+t^{2}\right) \omega-\frac{1}{12}\left(18-29 t+14 t^{2}-2 t^{3}\right) \\
R_{4 N}= & \frac{1}{36}\left(52-72 t+21 t^{2}\right) \omega^{2}+\frac{1}{180}\left(144-260 t+120 t^{2}-15 t^{3}\right) \omega \\
& -\frac{1}{360}\left(630-1164 t+725 t^{2}-180 t^{3}+15 t^{4}\right) \\
R_{5 N}= & \frac{1}{180}\left(264-510 t+280 t^{2}-45 t^{3}\right) \omega^{3}+\frac{1}{180}\left(120-264 t+170 t^{2}-40 t^{3}+3 t^{4}\right) \omega \\
& -\frac{1}{360}\left(720-1479 t+1094 t^{2}-365 t^{3}+55 t^{4}-3 t^{5}\right),
\end{aligned}
$$

when $N \geq 6$. The same sort of reasoning shows that

$$
(1+D)^{-2}=1+\omega(t) \sum_{p=1}^{N-1} u_{p N}(t) t^{p}(n+1)^{-p}
$$

in which $u_{p N}$ is independent of $n$ and $N$ and is bounded when $0 \leq t<\infty$ by a polynomial of degree $p-1$ in $t$ when $1 \leq p \leq N-2$, and $u_{N-1, N}$ is bounded when $0 \leq t \leq a$ by a polynomial of degree $N-2$ in $t$ whose coefficients are independent of $n$. (It is necessary to observe that $D \geq 0$.) In particular,

$$
\begin{aligned}
& u_{1 N}=-2, \quad u_{2 N}=3 \omega-\frac{1}{3}(4-3 t), \quad u_{3 N}=-4 \omega^{2}+(4-3 t) \omega-\frac{1}{3}\left(3-4 t+t^{2}\right), \\
& u_{4 N}=5 \omega^{3}-(8-6 t) \omega^{2}+\frac{1}{12}\left(52-72 t+21 t^{2}\right) \omega-\frac{1}{180}\left(144-260 t+120 t^{2}-15 t^{3}\right), \\
& \text { when } N \geq 6
\end{aligned}
$$


We next observe that

$$
E=t^{-2} \chi(t) R(1+D)^{-2}=\chi(t) \sum_{p=1}^{N} E_{p N}(t)(n+1)^{-p}
$$

in which $E_{p N}$ is independent of $n$ and $N$ and is bounded when $0 \leq t<\infty$ by a polynomial of degree $2 p-2$ in $t$ when $1 \leq p \leq N-1$. (The bound is not obvious when $p=1$; it is true because $E_{1 N}=t^{-1} R_{1 N}=\operatorname{coth} t-t^{-1}$.) Moreover, $E_{N N}$ is bounded when $0 \leq t \leq a$ by a polynomial of degree $2 N-2$ in $t$ whose coefficients are independent of $n$. In particular,

$$
\begin{gathered}
E_{2 N}=-3 \omega^{2}+\frac{1}{3}(10-9 t) \omega-\frac{1}{12}\left(15-20 t+6 t^{2}\right) \\
E_{3 N}=t\left\{4 \omega^{3}-(7-6 t) \omega^{2}+\frac{1}{6}\left(29-42 t+14 t^{2}\right) \omega-\frac{1}{6}\left(18-29 t+14 t^{2}-2 t^{3}\right)\right\} \\
E_{4 N}=t^{2}\left\{-5 \omega^{4}+(12-10 t) \omega^{3}-\frac{1}{12}\left(145-216 t+75 t^{2}\right) \omega^{2}\right. \\
+\frac{1}{60}\left(388-725 t+420 t^{2}-75 t^{3}\right) \omega \\
\left.\quad-\frac{1}{360}\left(630-1164 t+725 t^{2}-180 t^{3}+15 t^{4}\right)\right\} \\
E_{5 N}=t^{3}\left\{6 \omega^{5}-\frac{1}{3}(55-45 t) \omega^{4}+\frac{1}{3}\left(73-110 t+39 t^{2}\right) \omega^{3}\right. \\
-\frac{1}{60}\left(1094-2190 t+1375 t^{2}-270 t^{3}\right) \omega^{2} \\
+\frac{1}{180}\left(1479-3282 t+2555 t^{2}-825 t^{3}+93 t^{4}\right) \omega \\
\left.-\frac{1}{360}\left(720-1479 t+1094 t^{2}-365 t^{3}+55 t^{4}-3 t^{5}\right)\right\}
\end{gathered}
$$

when $N \geq 6$.

We state our next result in this chain of manipulations as follows.

LEMMA 5. There are functions $G_{p N}(t)$ such that

$$
(1+E)^{1 / 2}-1=\chi(t) \sum_{p=1}^{N} G_{p N}(t)(n+1)^{-p} .
$$

The function $G_{p N}$ is independent of $n$ and $N$ and is bounded when $0 \leq t<\infty$ by a polynomial of degree $2 p-2$ in $t$ when $1 \leq p \leq N-1$. The function $G_{N N}$ is bounded when $0 \leq t \leq a$ by a polynomial of degree $2 N-2$ in $t$ whose coefficients are independent of $n$. In particular,

$$
\begin{gathered}
G_{1 N}=\frac{1}{2} E_{1 N}, \quad G_{2 N}=\frac{1}{2} E_{2 N}-\frac{1}{8} \chi E_{1 N}^{2} \\
G_{3 N}=\frac{1}{2} E_{3 N}-\frac{1}{4} \chi E_{1 N} E_{2 N}+\frac{1}{16} \chi^{2} E_{1 N}^{3} \\
G_{4 N}=\frac{1}{2} E_{4 N}-\frac{1}{8} \chi\left(E_{2 N}^{2}+2 E_{1 N} E_{3 N}\right)+\frac{3}{16} \chi^{2} E_{1 N}^{2} E_{2 N}-\frac{5}{128} \chi^{3} E_{1 N}^{4} \\
G_{5 N}=\frac{1}{2} E_{5 N}-\frac{1}{4} \chi\left(E_{1 N} E_{4 N}+E_{2 N} E_{3 N}\right) \\
+\frac{3}{16} \chi^{2} E_{1 N}\left(E_{2 N}^{2}+E_{1 N} E_{3 N}\right)-\frac{5}{32} \chi^{3} E_{1 N}^{3} E_{2 N}+\frac{7}{256} \chi^{4} E_{1 N}^{5}
\end{gathered}
$$

when $N \geq 6$.

This follows by a now familiar reasoning from (6) and the identity,

$$
(1+E)^{1 / 2}-1=\sum_{k=1}^{N}\left\{\frac{\Gamma\left(k-\frac{1}{2}\right)(-1)^{k-1} \gamma_{k N}}{2 \pi^{1 / 2} k !}\right\} E^{k},
$$


in which $\gamma_{k N}=1$ when $1 \leq k \leq N-1$ and $\gamma_{N N}=\left(1+\theta_{4} E\right)^{-N+1 / 2}$, that is valid for some $\theta_{4}$ such that $0<\theta_{4}<1$. We need to observe that, as a consequence of Lemma $4,1+\theta_{4} E \geq 0.3492, \gamma_{N N} \leq(2.8367)^{N-1 / 2}$.

3. Proof of the theorem. We observe first that

$$
\begin{gathered}
\nu_{n}=\frac{4}{\pi} \int_{0}^{\delta}\left(1-x^{2}\right)^{-1} d x-F+G, \\
\nu_{n}=(2 / \pi) \log \{(1+\delta) /(1-\delta)\}-F+G,
\end{gathered}
$$

in which

$$
\begin{aligned}
F & =\frac{4}{\pi} \int_{0}^{\delta}\left(1-x^{2}\right)^{-1}\left[1-\left\{1-h_{n}^{2}(x)\right\}^{1 / 2}\right] d x \\
& =\frac{4}{\pi} \int_{0}^{\delta}\left(1-x^{2}\right)^{-1} h_{n}^{2}(x)\left[1+\left\{1-h_{n}^{2}(x)\right\}^{1 / 2}\right]^{-1} d x \\
G & =\frac{4}{\pi} \int_{\delta}^{1}\left(1-x^{2}\right)^{-1}\left\{1-h_{n}^{2}(x)\right\}^{1 / 2} d x
\end{aligned}
$$

We now show that $F$ is asymptotically negligible.

LEMMA 6. $F=O\left(a^{3} e^{-2 a}\right)=o\left\{(n+1)^{-N}\right\}$ for large $n$ and any $N$.

Here and throughout this section the constants implicit in the symbols $O$ and $o$ may depend on $N$, but are independent of $n$. Because $0 \leq h_{n}(x) \leq(n+1) x^{n}$,

$$
0<F<\frac{4(n+1)^{2}}{\pi\left(1-\delta^{2}\right)} \int_{0}^{\delta} x^{2 n} d x=\frac{4(n+1)^{2} \delta^{2 n+1}}{\pi\left(1-\delta^{2}\right)(2 n+1)}
$$

We observe when $n \geq 8$ that

$$
1-\delta^{2}=a^{-1}\left(2-a^{-1}\right) \geq 5 / 3 a, \quad 2(n+1) /(2 n+1) \leq 18 / 17,
$$

and

$$
\begin{aligned}
\delta^{2 n+1} & =\exp \left\{(2 n+1) \log \left(1-a^{-1}\right)\right\}<\exp \left\{-(2 n+1) a^{-1}\right\} \\
& =\exp \left(-2 a+a^{-1}\right) \leq e^{1 / 3} e^{-2 a} .
\end{aligned}
$$

The assertion of the lemma is now apparent.

It we make the substitution $x=1-t /(n+1)$ in $G$ and use Lemma 2 , we see that

$$
\begin{gathered}
G=\frac{2}{\pi} \int_{0}^{a}\left[\{2(n+1)-t\}^{-1}+t^{-1}\right] d t+A_{0}-H+I+J+K, \\
G=(2 / \pi) \log \{a /(1+\delta)\}+A_{0}-H+I+J+K,
\end{gathered}
$$

in which $A_{0}$ is the constant defined in (3) and

$$
\begin{gathered}
H=\frac{2}{\pi} \int_{0}^{a}\{2(n+1)-t\}^{-1}\left\{1-\left(1-t^{2} \operatorname{csch}^{2} t\right)^{1 / 2}\right\} d t \\
I=\frac{2}{\pi} \int_{0}^{a}\left(1-t^{2} \operatorname{csch}^{2} t\right)^{1 / 2} t^{-1}\left\{(1+E)^{1 / 2}-1\right\} d t \\
J=\frac{2}{\pi} \int_{0}^{a}\{2(n+1)-t\}^{-1}\left(1-t^{2} \operatorname{csch}^{2} t\right)^{1 / 2}\left\{(1+E)^{1 / 2}-1\right\} d t \\
K=\frac{2}{\pi} \int_{a}^{\infty}\left\{1-\left(1-t^{2} \operatorname{csch}^{2} t\right)^{1 / 2}\right\} t^{-1} d t
\end{gathered}
$$


We now show that $K$ is asymptotically negligible.

LEMMA 7. $K=O\left(a e^{-2 a}\right)=o\left\{(n+1)^{-N}\right\}$ for large $n$ and any $N$.

It is obvious that $0 \leq 1-(1-s)^{1 / 2} \leq s$ when $0 \leq s \leq 1$. Therefore,

$$
0<K<\frac{2}{\pi} \int_{a}^{\infty} t \operatorname{csch}^{2} t d t=O\left(a e^{-2 a}\right)
$$

An asymptotic expansion for $H$ can be inferred from the following lemma.

LEMMA 8. If $H_{p}, L$ and $M$ are defined so that

$$
\begin{gathered}
H_{p}=\left(2^{1-p} \pi^{-1}\right) \int_{0}^{\infty}\left\{1-\left(1-t^{2} \operatorname{csch}^{2} t\right)^{1 / 2}\right\} t^{p-1} d t \\
L=\sum_{p=1}^{N-1} 2^{1-p} \pi^{-1}(n+1)^{-p} \int_{a}^{\infty}\left\{1-\left(1-t^{2} \operatorname{csch}^{2} t\right)^{1 / 2}\right\} t^{p-1} d t \\
H=\sum_{p=1}^{N-1} H_{p}(n+1)^{-p}-L+M
\end{gathered}
$$

then $L=O\left(e^{-2 a}\right)$ and $M=O\left\{(n+1)^{-N}\right\}$ for large $n$.

The lemma follows from the definition of $H$, and Taylor's Theorem in the form

$$
(1-C)^{-1}=\sum_{p=0}^{N-2} C^{p}+C^{N-1}\left(1+\theta_{5} C\right)^{-N}, \quad 0<\theta_{5}<1,
$$

if we observe that $1-\theta_{5} C \geq 1-C \geq 1-1 / 2 a \geq 5 / 6$ when $n \geq 8$. In fact, $0<M<(6 / 5)^{N} H_{N}(n+1)^{-\bar{N}}$. Moreover,

$$
\begin{aligned}
0 & <L<\sum_{p=1}^{N-1} 2^{1-p} \pi^{-1}(n+1)^{-p} \int_{a}^{\infty} t^{p+1} \operatorname{csch}^{2} t d t \\
& =\sum_{p=1}^{N-1}(n+1)^{-p} O\left(a^{p+1} e^{-2 a}\right)=\sum_{p=1}^{N-1} O\left(a^{1-p} e^{-2 a}\right)=O\left(e^{-2 a}\right) .
\end{aligned}
$$

An asymptotic expansion for $I$ can be inferred from the following lemma.

LEMMA 9. If $I_{p}, S$ and $T$ are defined so that

$$
\begin{gathered}
I_{p}=\frac{2}{\pi} \int_{0}^{\infty} t^{3}\left(1-t^{2} \operatorname{csch}^{2} t\right)^{-1 / 2} \operatorname{csch}^{2} t G_{p N}(t) d t \\
S=\frac{2}{\pi} \sum_{p=1}^{N-1}(n+1)^{-p} \int_{a}^{\infty} t^{3}\left(1-t^{2} \operatorname{csch}^{2} t\right)^{-1 / 2} \operatorname{csch}^{2} t G_{p N}(t) d t \\
T=\frac{2}{\pi}(n+1)^{-N} \int_{0}^{a} t^{3}\left(1-t^{2} \operatorname{csch}^{2} t\right)^{-1 / 2} \operatorname{csch}^{2} t G_{N N}(t) d t
\end{gathered}
$$

then $I_{p}$ is independent of $n$ and $N$ when $1 \leq p \leq N-1, S=O\left(a e^{-2 a}\right)$ and $T=O\left\{(n+1)^{-N}\right\}$ for large $n$, and

$$
I=\sum_{p=1}^{N-1} I_{p}(n+1)^{-p}-S+T
$$


The identity (11) is an immediate consequence of Lemma 5. Moreover, $G_{p N}(t)=$ $O\left(t^{2 p-2}\right)$, uniformly in $n$ when $n \geq 8$, for large $t$, if $1 \leq p \leq N-1$, so that

$$
\begin{aligned}
S & =\sum_{p=1}^{N-1}(n+1)^{-p} \int_{a}^{\infty} O\left(t^{2 p+1} e^{-2 t}\right) d t \\
& =\sum_{p=1}^{N-1}(n+1)^{-p} O\left(a^{2 p+1} e^{-2 a}\right)=O\left(a e^{-2 a}\right) .
\end{aligned}
$$

Finally, the existence of the polynomial bound for $G_{N N}$ described in Lemma 5 implies that $T=O\left\{(n+1)^{-N}\right\}$.

If we combine Lemma 5 and (10) we find that

$$
\frac{(1+E)^{1 / 2}-1}{(n+1)(1-C)}=\chi(t) \sum_{p=2}^{N} Q_{p N}(t)(n+1)^{-p}
$$

in which $Q_{p N}$ is independent of $n$ and $N$ and is bounded when $0 \leq t<\infty$ by a polynomial of degree $2 p-4$ in $t$, when $2 \leq p \leq N-1$, and $Q_{N N}$ is bounded when $0 \leq t \leq a$ by a polynomial of degree $2 N-4$ in $t$ whose coefficients are independent of $n$. In particular,

$$
\begin{aligned}
Q_{2 N} & =\frac{1}{2} E_{1 N}, \quad Q_{3 N}=\frac{1}{2} E_{2 N}+\frac{1}{4} t E_{1 N}-\frac{1}{8} \chi E_{1 N}^{2} \\
Q_{4 N}=\frac{1}{2} E_{3 N}+ & \frac{1}{4} t E_{2 N}+\frac{1}{8} t^{2} E_{1 N}-\frac{1}{4} \chi\left(E_{2 N}+t E_{1 N} / 4\right) E_{1 N}+\frac{1}{16} \chi^{2} E_{1 N}^{3} \\
Q_{5 N}= & \frac{1}{2} E_{4 N}+\frac{1}{4} t E_{3 N}+\frac{1}{8} t^{2} E_{2 N}+\frac{1}{16} t^{3} E_{1 N} \\
& -\frac{1}{8} \chi\left(E_{2 N}^{2}+2 E_{1 N} E_{3 N}+t E_{1 N} E_{2 N}+t^{2} E_{1 N}^{2} / 4\right) \\
& +\frac{1}{16} \chi^{2} E_{1 N}^{2}\left(3 E_{2 N}+t E_{1 N} / 2\right)-\frac{5}{128} \chi^{3} E_{1 N}^{4}
\end{aligned}
$$

when $N \geq 6$. An argument almost identical to that used in the proof of Lemma 9 now suffices to establish the following result.

LEMMA 10. If $J_{p}, U$ and $V$ are defined so that

$$
\begin{gathered}
J_{p}=\pi^{-1} \int_{0}^{\infty} t^{4}\left(1-t^{2} \operatorname{csch}^{2} t\right)^{-1 / 2} \operatorname{csch}^{2} t Q_{p N}(t) d t \\
U=\pi^{-1} \sum_{p=2}^{N-1}(n+1)^{-p} \int_{a}^{\infty} t^{4}\left(1-t^{2} \operatorname{csch}^{2} t\right)^{-1 / 2} \operatorname{csch}^{2} t Q_{p N}(t) d t \\
V=\pi^{-1}(n+1)^{-N} \int_{0}^{a} t^{4}\left(1-t^{2} \operatorname{csch}^{2} t\right)^{-1 / 2} \operatorname{csch}^{2} t Q_{N N}(t) d t
\end{gathered}
$$

then $J_{p}$ is independent of $n$ and $N$ when $2 \leq p \leq N-1, U=O\left(e^{-2 a}\right)$ and $V=O\left\{(n+1)^{-N}\right\}$ for large $n$, and

$$
J=\sum_{p=2}^{N-1} J_{p}(n+1)^{-p}-U+V
$$

If we combine (7), (8), (9), (11) and (12), we see that

$$
\nu_{n}=\frac{2}{\pi} \log (n+1)+A_{0}+\sum_{p=1}^{N-1} A_{p}(n+1)^{-p}+O\left\{(n+1)^{-N}\right\},
$$


in which $A_{p}=-H_{p}+I_{p}+J_{p}$ (the hitherto undefined quantity $J_{1}=0$ ). This establishes the existence of the asymptotic expansion (5) of the Theorem, and prescribes the coefficients $A_{p}$. There remains only the straightforward, although tedious, verification of the specific results for $A_{1}, A_{2}, A_{3}, A_{4}$ and $A_{5}$ stated in the Theorem, based on the definitions of $H_{p}, I_{p}$ and $J_{p}$ given in Lemmas 8, 9 and 10. This verification is facilitated by the reduction formula

$$
\begin{aligned}
\int_{0}^{\infty} t^{2 q+p+1}\left(1-t^{2} \operatorname{csch}^{2} t\right)^{-q-1 / 2}(t \operatorname{coth} t-1) Z(t) \operatorname{csch}^{2 q+2} t d t \\
=\int_{0}^{\infty} t^{2 q+p-1}\left(1-t^{2} \operatorname{csch}^{2} t\right)^{-q+1 / 2} Z_{1}(t) \operatorname{csch}^{2 q} t d t
\end{aligned}
$$

valid if $1 \leq q, Z(t)$ is continuously differentiable on $(0, \infty)$, there is a positive integer $m$ such that $Z(t)=O\left(t^{m}\right)$ and $Z^{\prime}(t)=O\left(t^{m}\right)$ for large $t, Z(t)=O\left(t^{2 q-p}\right)$ and $Z^{\prime}(t)=O\left(t^{2 q-p}\right)$ for small $t$, and

$$
Z_{1}(t)=\left\{(2 q+p-2 q t \operatorname{coth} t) Z(t)+t Z^{\prime}(t)\right\} /(2 q-1) .
$$

Kahaner [10] has tabulated seven decimal place values of $\nu_{n}$ when $n=1(1) 100$. The expansion (5), terminated after the term for which $p=2$ (or $p=3$ ), produces seven decimal place values that either agree with or exceed by at most $10^{-7}$ those of Kahaner when $n \geq 30$. Inclusion of the term for which $p=4$ (and $p=5$ ) produces the same agreement when $n \geq 8$. The error when $n=1$ is only 0.0008575 . (There is a typographical error in the value $\nu_{98}$ reported by Kahaner. The correct value is 3.5510552.)

\section{REFERENCES}

1. M. Kac, On the average number of real roots of a random algebraic equation, Bull. Amer. Math. Soc. 49 (1943), 314-320.

2. B. R. Jamrom, The average number of real zeros of random polynomials, Soviet Math. Dokl. 13 (1972), 1381-1383.

3. Ja. B. Jamrom, On the average number of real roots of a random algebraic polynomial, Vestnik Leningrad. Univ. Ser. Math. Meh. 4 (1971), no. 19, 152-156.

4. Y. J. Wang, Bounds on the average number of real roots of a random algebraic equation, Chinese Ann. Math. Ser. A. 4 (1983), 601-605.

5. D. C. Stevens, The average and variance of the number of real zeros of random functions, $\mathrm{Ph} . \mathrm{D}$. dissertation, New York Univ., 1965.

6. J. E. Wilkins, Jr., An upper bound for the expected number of real zeros of a random polynomial, J. Math. Anal. Appl. 42 (1973), 569-577.

7. Z. H. Luo, The average number of real roots of a random algebraic equation, Chinese Ann. Math. 1 (1980), 541-544.

8. M. J. Christensen and M. Sambandham, An improved lower bound for the expected number of real zeros of a random polynomial, Stochastic Anal. Appl. 2 (1984), 431-436.

9. Z. M. Yu, Bounds on the average number of real roots for a class of random algebraic equations, J. Math. Res. Exposition 2 (1982), 81-85.

10. D. K. Kahaner, Some computations of expected number of real zeros of random polynomials, J. Math. Anal. Appl. 48 (1974), 780-784.

4800 S. LAKe Shore Drive, \# 1501N, Chicago, Illinois 60615 